Category: Teaching \& Learning at times of uncertainty

\title{
The design and development of a module applied to lesson plan in a blended approach
}

\author{
Hassan, S. \\ International Medical University, Malaysia
}

\section{Background:}

The design of lesson plan applied to a module is a hybrid conceptual model developed to meet the challenges of COVID-19 crisis with features of being completely transferable to the online learning experience. The instructional strategies are based on Gagne's 9 events to meet the learning outcome. The model aims to provide a values-driven learning experience in a personalised learning environment with control over time, pace, path, and place of learning and self-assessment that helps to develop metacognitive skills among students. The objective of the study is to meet the challenges of restrictions to F2F instructions and continuing the teaching and learning experience with a personalised online learning approach.

\section{Method:}

The design and development of a lesson plan applied to a module are based on a blended learning approach to make a face to face topic converted to a lesson plan and becoming a readily available learning material for online delivery.

\section{Conclusion:}

The model offers a value-driven students' experience in a personalised learning environment with control over time, pace, path, and place of learning and self-assessment to help develop the metacognitive approach.

Keyword: Lesson Plan 